\section{Medical council funds both clinical and basic research}

SIR - Your Editorial “Brown's budget briefing” (Nature 440, 581; 2006) implies that the UK Medical Research Council (MRC) is responsible only for basic biomedical research. In fact, MRC scientists invented randomized clinical trial methodology in the 1940s, and the MRC remains the largest UK public funder of trials. It has supported a multitude of trials including the Heart Protection Study (demonstrating the value of statins) and the CRASH trial (showing that the treatment of head-injury victims with corticosteroids is actually dangerous), both of which were highlighted in the budget statement.

The MRC is internationally recognized for supporting research that has underpinned the twentieth-century revolution in basic biomedicine. But its contribution to clinical research has been equally significant. Not just its trials and epidemiology (including discovery of the link between smoking and cancer), but ground-breaking work in such areas as vitamins, viruses, penicillin, vaccines, magnetic resonance imaging and antibody drugs.

Three years ago, the MRC made a strategic commitment to increase its investment in translational, clinical and public-health research. The planned creation of a new, single fund for health research is an opportunity to strengthen further the pipeline from discovery to better treatment and prevention. But, as you point out, maintaining the independence of scientific judgement that has delivered so well in the past will be vital.

Colin Blakemore

Medical Research Council,

20 Park Crescent, London W1B 1AL, UK

\section{Exaggerating one's success is rife in Chinese academia}

SIR - Your News in Brief story "Fantasy reference list leads to the sack" (Nature 440,728 ; 2006) reports on the dismissal of Hui Liu, assistant dean of the medical school at Tsinghua University in Beijing. But exaggerating the scientific significance of one's research is even more harmful to the reputation of China's academic communities than faking a $\mathrm{CV}$ or résumé.

The Chinese Academy of Sciences (CAS) plays an important role in setting the course for scientific and technological development. Its members are scientists, engineers and technologists, who are eminent scholars with a strong influence on China's science and technology policy. CAS membership is a high honour, but reading the exaggerated biographies of some members and candidates, one feels some of them deserve Nobel prizes. Ordinary research results are often portrayed as extraordinary scientific achievements.

Partly being driven by the country's 'knowledge innovation' policy, Chinese scientists have made it a priority to pursue publications in journals with high impact factors. The peer pressure on postgraduate students, as well as on CAS members, has made exaggeration of scientific achievements and overstatement of personal talent quite common in China. But ill-minded overexaggeration, bragging, plagiarizing and fraud are morally wrong and should be legally challenged, in China or anywhere else.

\section{Zheng Huang}

Radiation Oncology Department, University of Colorado at Denver and Health Sciences Center, Aurora, Colorado 80045, USA

\section{Allergy test might have avoided drug-trial disaster}

SIR - Your News story "Can super-antibody drugs be tamed?" (Nature 440, 855-856; 2006), on the TGN1412 anti-CD28 antibody drug and the fallout from this near-fatal clinical trial, lacks a simple interpretation of the data based on common sense.

Accounts of the clinical trial describe how, following intravenous infusion of the antibody, the volunteers were clawing off their clothes, swelling with obvious oedema, and starting to suffer pain and panic within minutes. The developer of TGN1412, Thomas Hunig, links this disabling "cytokine storm" to T-cell activation. But such a reaction would take hours.

In fact, these volunteers could have been victims of the release of preformed mediators, of the types usually found within the granules of cells of an allergic response. As any subject of an anaphylactic response can testify myself included - the response is immediate, with similar symptoms to those experienced by the volunteers in the TGN1412 trial.

The literature suggests that CD28 can be expressed by cells of the allergic response (M. Tashiro et al. J. Immunol. 158, 2382$2389 ; 1997)$, and the real disaster was that no tests were carried out for the possibility of allergic reactions before TGN1412 was released into the blood stream. A simple 'weal and flare' allergic-response test could have easily alerted the clinicians to a potential allergic reaction. But reports have suggested that no such tests were performed. The first human trial with this drug was the intravenous infusion that led to such catastrophic responses in these volunteers. John H. Weis

Pathology Department, University of Utah School of Medicine, 30 North 1900 East 5C124 SOM, Salt Lake City, Utah 84132, USA

\section{Keeping an eye on privacy issues with geospatial data}

SIR - In their Commentary "Mapping disaster zones" (Nature 439, 787-788; 2006), Illah Nourbakhsh and colleagues rightly say that the global distribution of spatial data, especially high-resolution aerial photography, may raise issues of privacy violation. We at Louisiana State University used Google Earth extensively in the Emergency Operation Center to facilitate search-and-rescue missions immediately after Hurricane Katrina. There is no denying the utility this system provided. But, in the months after the hurricane, we used the markings left by these same search-andrescue teams to highlight the danger of using geospatial technology.

A map displaying body-recovery locations in the New Orleans area was cut out of a local newspaper and turned into a Geographic Information System (GIS) layer, using census boundary information to guide the transfer as no streets and limited city landmarks were on the original. Once in the GIS, the central coordinates of each mortality 'dot' were extracted. A field team using the Global Positioning System checked the locations where bodies had been found against the search-and-rescue markings sprayed on houses. In many cases the dots, which on the map covered approximately one-anda-half city blocks, revealed the location to be within an area of two or three neighbouring houses, with the exact house being identified in some cases.

What would have happened if a scientist had presented HIV cases, or nesting sites of an endangered species, on a similar city display - relying on an oversized point symbol to mask the real-world location? There is scant guidance for scientists to help preserve spatial confidentiality. Current standards often address only the required size for denominator populations in a thematic map display. Unless we in academia take the lead in expanding and enforcing a more rigid set of spatial display rules, especially for point data, we run the risk of an over-zealous tightening of data release and a protracted battle to again persuade those in power that a map can be used for the good of society. Andrew Curtis ${ }^{\star}$, Jacqueline W. Mills Michael Leitner*

*Department of Geography and Anthropology, Louisiana State University, Baton Rouge,

Louisiana 70803, USA

†LSU GIS Clearinghouse, CADGIS Research Lab, Louisiana State University, Baton Rouge, Louisiana 70803, USA

Contributions to Correspondence may be submitted to corres@nature.com.They should be no longer than $\mathbf{5 0 0}$ words, and ideally shorter. 